\title{
A Model-Based Substructuring Method for Local Damage Detection of Structure
}

\author{
Eun-Taik Lee ${ }^{1}$ and Hee-Chang Eun ${ }^{2}$ \\ ${ }^{1}$ Department of Architectural Engineering, Chung-Ang University, 84 Heukseok-ro, Dongjak-Gu, \\ Seoul 156-756, Republic of Korea \\ 2 Department of Architectural Engineering, Kangwon National University, Hyoja 2 Dong, \\ Chuncheon 200-701, Republic of Korea \\ Correspondence should be addressed to Hee-Chang Eun; heechang@kangwon.ac.kr
}

Received 16 September 2012; Accepted 14 March 2013; Published 11 February 2014

Academic Editor: Gyuhae Park

Copyright (C) 2014 E.-T. Lee and H.-C. Eun. This is an open access article distributed under the Creative Commons Attribution License, which permits unrestricted use, distribution, and reproduction in any medium, provided the original work is properly cited.

\begin{abstract}
It is impractical to collect the full set of data in finite element model for investigating the structural performance. This study presents a model-based substructuring method to examine it by the data measured at a few positions. Dividing a global structure into several substructures and regarding the frequency response functions (FRFs) measured at the interfaces between the adjacent substructures as the constraints, the constraint forces at the substructure nodes are calculated to decide the resultant responses. The damageexpected substructure is traced by the distribution curve of constraint forces. Modeling the damage-expected substructure subjected to the predicted constraint forces and expanding the displacement data measured at several locations in the substructure, the local damage is detected by the displacement curvature method. A numerical application illustrates the validity of the proposed method.
\end{abstract}

\section{Introduction}

Structural health monitoring has been receiving a growing amount of interest from researchers in diverse fields of engineering. The static or dynamic strain energy of an intact system is changed if damage in the system exists. The damage exists at the element that the variation in the static or dynamic strain energy is observed. Damage detection techniques are performed by collecting necessary data by both experimental and numerical means and comparing the response difference between the undamaged and damaged states.

Most damage detection methods require knowing fundamental information for a structure without damage. The test data should be compared with the baseline data to identify and locate damage. One of the damage detection methods takes the process to isolate the damage-expected substructure from a global structure and to identify the local damage in it. If the deterioration of structural performance in the global structure is expected, it is divided into several substructures and the damage-expected substructure is predicted by the proper damage detection method. The isolated damage-expected substructure is investigated to detect the damage within the tiny region.

Hou et al. [1] presented the substructure isolation method (SIM) using two steps of the construction of isolated substructure and the damage identification of substructure. The method extended the SIM in the time domain to that in the frequency domain. The damage is detected by comparing the natural frequencies of the isolated substructure and FEM model. Decomposing a structural flexibility matrix into a matrix representation of the connectivity, Yang [2] presented a structural damage identification method based on flexibility disassembly. Yang and Sun [3] proposed a staticbased damage detection method using the flexibility disassembly technique and a damage localization vector. Yuen and Katafygiotis [4] proposed a probabilistic substructure identification approach not to require any information regarding the input. Law et al. [5] identified the coupling forces between substructures from the acceleration response and the structural damage from the coupling forces based on dynamic sensitivity analysis. 


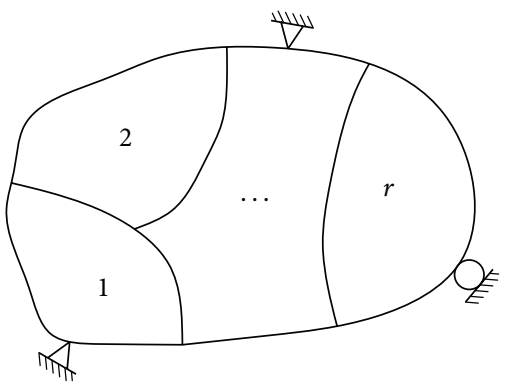

(a)

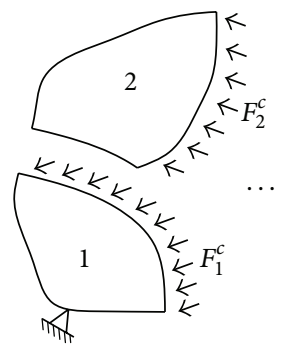

(b)

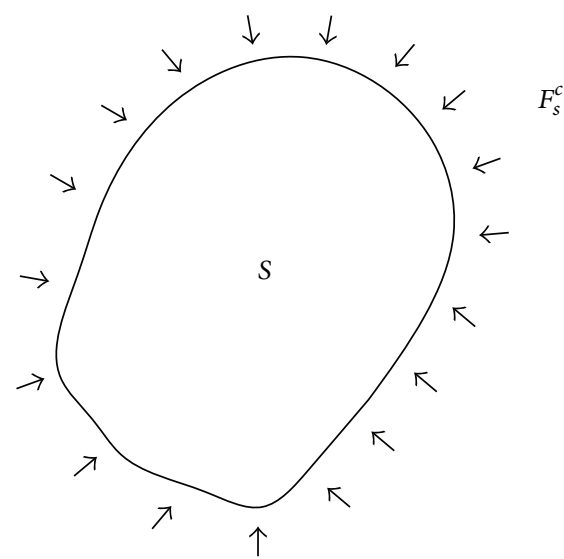

(c)

FIGURE 1: Substructuring of a global structure: (a) a global structure, (b) decomposition of $r$ substructures, and (c) sth substructure subjected to external and constraint forces.

Xing and Mita [6] proposed a substructure approach to divide a complete structure into several substructures so that damage detection processes can be independently conducted on each substructure. Based on sequential application of the extended Kalman estimator for the extended state vector and the least squares estimation for the unknown inputs, Xing et al. [7] proposed a substructure approach to detect structural local damage through the degradation of the stiffness. Based on this structural damage detection to detect structural local damage in complex structures, Lei et al. [8] developed a two-stage damage detection strategy to the ASCE SHM benchmark building. Hou et al. [9] proposed a frequency domain method of substructure identification for local health monitoring consisting of the construction of isolated substructure and damage identification of substructure. Adding virtual supports on the substructure interface using the FRF of measured local responses of the global structure and isolating the substructures, they proposed a substructuring method. Qian et al. [10] proposed a sensor region-based damage detection approach based on the damage locating vectors method to detect the damage regions of the substructure. Zhang and Johnson [11] introduced a substructure identification method for shear structures by dividing them into many substructures. All story parameters are estimated utilizing the cross-power spectral densities between structural floor accelerations and a reference response. They proposed a substructure method based on the identification error analysis. Law et al. [12] identified the coupling forces between the substructures from the acceleration response of a structure and detected structural damage from the identified coupling forces based on dynamic sensitivity analysis. Weng et al. [13] provided the sensitivity-based model updating method by iteratively adjusting the parameter matrices and a substructuring method to predict the Eigen solutions.

Most of the substructuring-based damage detection methods determined the coupling forces utilizing the compatibility conditions between adjacent substructures and performed the sensitivity analysis of modal parameters. The coupling forces can be estimated in the time domain and in the frequency domain. The FRF data in the frequency domain provide more information than modal data, as the latter are extracted from a very limited frequency range related to resonance. This study used the measured FRF data in the frequency domain.

The constraint forces to satisfy the displacement data measured at the interface nodes between adjacent substructures are calculated synthesizing the FRF matrix at the intact state and the measured data. The damage-expected substructure is predicted from the abrupt change of the constraint forces in the frequency domain. Then isolating the damageexpected substructure only subjected to the constraint forces and expanding a few measurement data, the displacement responses of the isolated substructure are estimated. The local damage in the substructure is found by the damage detection algorithm such as the displacement curvature curve. The proposed method has a merit to utilize less measurement data than the total number of dofs of finite element model. The validity of the proposed method is illustrated in a numerical experiment.

\section{Local Damage Detection by Substructuring Approach}

The damage detection in a complicated structure becomes less sensitive with the increase of the distance between the damage and measurements. Because a complicated structure is composed of several substructures, the local damage can be traced by gradually closing from a global structure to the damage-expected range. This substructuring method is performed by estimating the constraint forces at the nodes between the adjacent substructures and expanding a few measurement data to the number of dofs in finite element model. The damage is detected by the estimated response data or their modification.

Assume that a structure consists of $r$ independent substructure as shown in Figure 1(a). Each substructure has $n_{j}$ dofs $(j=1,2, \ldots, r)$. Assuming the total number of dofs for 


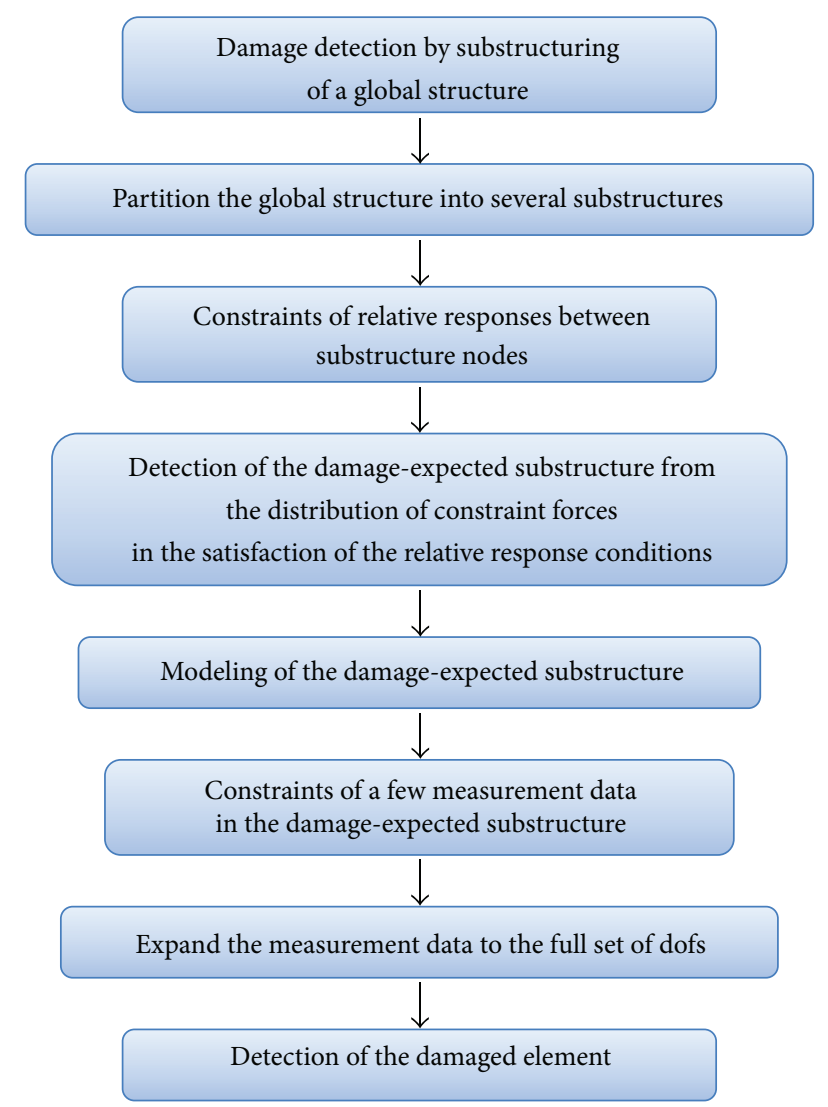

FIGURE 2: Summary of the proposed substructuring method for damage detection.

the $r$ substructures as $n p$ dofs and expressing the overlapped dofs at $l$ different joints as $n l$, the dofs of the global structure, $n$, can be written as

$$
n=n p-n l,
$$

where $n p=\sum_{j=1}^{r} n_{j}$ and $n l=\sum_{i=1}^{l} n_{i}$.

The dynamic behaviour of the global structure at intact state can be described by the equations of motion as

$$
\mathbf{M} \ddot{\mathbf{u}}+\mathbf{C} \dot{\mathbf{u}}+\mathbf{K u}=\mathbf{F}(t),
$$

where $\mathbf{M}, \mathbf{C}$, and $\mathbf{K}$ denote the $n \times n$ analytical mass, damping, and stiffness matrices. And $\mathbf{F}(t)$ is the $n \times 1$ excitation vector.

Inserting $\mathbf{u}=\widehat{\mathbf{U}} e^{j \Omega t}$ and $\mathbf{F}=\widehat{\mathbf{F}} e^{j \Omega t}$ into (2) and expressing it in the frequency domain, it follows that

$$
\left(\mathbf{K}-\Omega^{2} \mathbf{M}+j \Omega \mathbf{C}\right) \widehat{\mathbf{U}}(\Omega)=\widehat{\mathbf{F}}(\Omega),
$$

where $\Omega$ denotes the excitation frequency, $\widehat{\mathbf{F}}$ is an external force vector with an element being unit and all other elements zeros, and $j=\sqrt{-1}$. At an arbitrary frequency, the dynamic equation of (3) becomes the static equation of the same form as the equilibrium equation. Using the receptance-type matrix of the structure $\mathbf{H}(\Omega)$, the equation of motion for the initial structure in the frequency domain is expressed by

$$
\widehat{\mathbf{U}}(\Omega)=\mathbf{H}(\Omega) \widehat{\mathbf{F}}(\Omega),
$$

where $\mathbf{H}(\Omega)=\mathbf{B}^{-1}(\Omega)$ is the FRF matrix of the finite element model, whose elements can be the receptances and $\mathbf{B}(\Omega)=$ $\left(\mathbf{K}-\Omega^{2} \mathbf{M}+j \Omega \mathbf{C}\right)$. The element $H_{i, j}$ in the matrix $\mathbf{H}$ indicates a displacement response at station $i$ and a disturbing force at station $j$.

The dynamic responses corresponding to the full set of dofs of the global structure are calculated from (4). However, it is impractical to measure all responses of the full dofs of the global structure to investigate the structural performance. In order to reduce such impractical calculations, this work traces the local damage by closing to the region that the damage is expected from the global structure. The substructuring is carried out for this purpose.

This work is composed of two steps. The first step detects the damage-expected substructure of $r$ substructures. The global structure of $n$ dofs is modified to the dynamic system of the $n l$ overlapped dofs to partition the $r$ substructures.

Consider

$$
\widehat{\mathbf{U}}_{R}(\Omega)=\widehat{\mathbf{H}}_{R}(\Omega) \widehat{\mathbf{F}}_{R}(\Omega),
$$

where the subscript $R$ represents the dynamic system of $n l$ overlapped dofs.

Let us assume that unknown damage exists in the structure. If the initial FRF matrix of the structure corresponding to the $n l$ dofs is given, the structural performance of the damage-expected substructure can be evaluated by the constraint forces. The constraints are expressed by the displacements relative to the reference displacement of the stationary substructure node. They are written by

$$
\mathbf{A U}_{R}=\mathbf{0},
$$

where $\mathbf{A}$ denotes the $n f \times n l(n f<n l)$ matrix and $n f$ is the number of the constraints. And $\mathbf{U}_{R}$ is the actual dynamic response vector at the damage-expected state.

The dynamic equation of (5) should be modified due to the damage presence and combined with (6) to describe the response of the damaged structure. It is derived based on the generalized inverse method [14] introduced in Appendix A as

$$
\mathbf{U}_{R}=\widehat{\mathbf{U}}_{R}+\delta \widehat{\mathbf{U}}_{R}=\widehat{\mathbf{U}}_{R}-\widehat{\mathbf{H}}_{R}^{0.5}\left(\mathbf{A} \widehat{\mathbf{H}}_{R}^{0.5}\right)^{+} \mathbf{A} \widehat{\mathbf{U}}_{R},
$$

where $\widehat{\mathbf{U}}_{R}$ is the dynamic response vector of the substructures, $\delta \widehat{\mathbf{U}}_{R}$ denotes the variation in the response, each element of $\widehat{\mathbf{H}}_{R}$ denotes the absolute value of the complex number, and "+" represents the Moore-Penrose inverse. The second term in the right-hand side of (7) denotes the displacement variation due to the constraints. And the constraint forces $\mathbf{F}_{R}^{c}$ to cause the response variation are derived as

$$
\mathbf{F}_{R}^{c}=-\widehat{\mathbf{H}}_{R}^{-0.5}\left(\mathbf{A} \widehat{\mathbf{H}}_{R}^{0.5}\right)^{+} \mathbf{A} \widehat{\mathbf{U}}_{R} .
$$

The damage leads to the increase in displacement variation as well as constraint force. The damage exists in the neighborhood of the location to require the large constraint force as shown in (6) and (7). The damage-expected substructure can be detected from the abrupt change of the constraint 


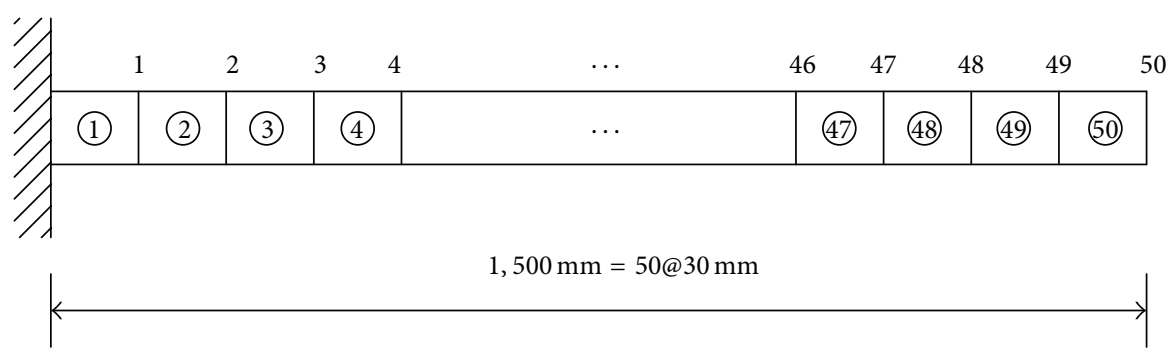

(a)

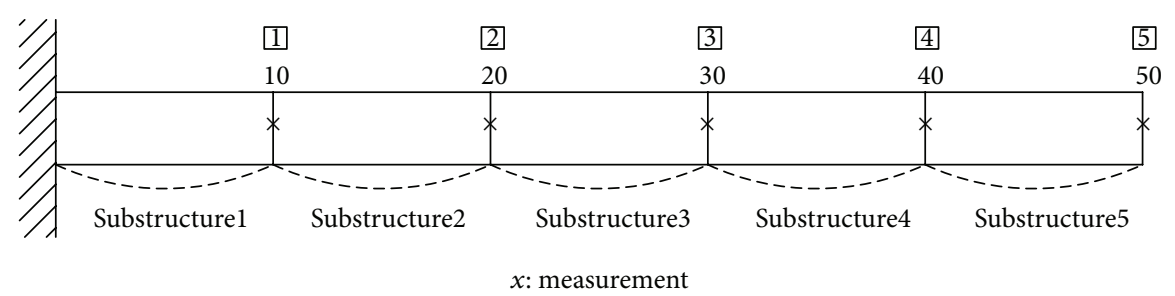

(b)

FIGURE 3: Substructuring of a cantilevered beam: (a) a cantilevered beam and (b) decomposition of a cantilevered beam.

forces or the displacement variation. At the next step, the damaged element in the damage-expected substructure is traced from the isolated substructure model.

The isolated sth substructure in Figure 1(c) is depicted as a model subjected to the constraint forces established at the previous step. The dynamic equation of the sth substructure at the intact state can be written by

$$
\widehat{\mathbf{U}}_{s}(\Omega)=\mathbf{H}_{s}(\Omega) \mathbf{F}_{s}(\Omega),
$$

where $\mathbf{F}_{s}$ denotes the $n z \times 1$ force vector of $\mathbf{F}_{s}(\Omega)=$ $\left[\begin{array}{lllll}\widehat{\mathbf{F}}_{s-1}^{c} & 0 & \cdots & 0 & 0\end{array}\right]^{T}, n z$ is the number of dofs of the $s$ th substructure, and $\widehat{\mathbf{F}}_{s-1}^{c}$ represents the constraint force vector at the interfaces to contact the sth substructure with the other substructures.

Assuming the measurements are performed at fewer locations than the total number of dofs of the isolated substructure, the data should be expanded. The responses data measured at $l(l<n z)$ locations are utilized as constraints to describe the constrained dynamic responses of the sth substructure. The measured data are expressed by

$$
\mathbf{D}_{l \times n z} \mathbf{U}_{s}(\Omega)=\mathbf{w},
$$

where $\mathbf{D}$ is the $l \times n z$ Boolean matrix to define the measurement locations, $\mathbf{w}$ is the measured displacement vector, and $\mathbf{U}_{s}$ represents the actual displacement response vector of the sth substructure.

Combining the dynamic equation of (9) and the constraint equations of (10), the constrained dynamic equation [14] introduced in Appendix A can be written by

$$
\mathbf{U}_{s}=\widehat{\mathbf{U}}_{s}+\mathbf{H}_{s}^{0.5}\left(\mathbf{D H}_{s}^{0.5}\right)^{+}\left(\mathbf{w}-\mathbf{D} \widehat{\mathbf{U}}_{s}\right) \text {. }
$$

The second term in the right-hand side of (11) denotes the displacement variation due to damage. The local damage is detected by investigating the dynamic responses estimated by (11). The abrupt displacement variation in the neighborhood of the damage is expected. However, the displacement variation does not provide the definite information on the damage and we utilize the modified form of the displacements such as the displacement curvature. The proposed substructuring method is summarized in Figure 2 and its applicability is evaluated in the following example.

\section{Damage Detection of a Cantilevered Beam}

Let us consider the damage detection of a cantilevered beam in Figure 3(a). The nodal points and the members are numbered as shown in the figure. Assuming simplest BernoulliEuler plane beam element, the beam finite elements are obtained by subdividing beam members longitudinally. Each node has two dofs of transverse displacement and slope. However, we considered only the parameter matrices corresponding to the transverse displacements of the beam because the measurement of the slope is nearly impossible. The beam of $1.5 \mathrm{~m}$ length was modeled by 50 beam elements that the length of each is $30 \mathrm{~mm}$. The beam has elastic modulus of $1.95 \times 10^{5} \mathrm{MPa}$ and unit mass of $7,860 \mathrm{~kg} / \mathrm{m}^{3}$. Its gross cross section was established as $40 \mathrm{~mm} \times 3 \mathrm{~mm}$, and the damaged cross-section of $10 \%$ section loss located at the element 14. This numerical work assumed the stiffness degradation, the stiffness proportional Rayleigh damping, and the invariant mass despite the damage.

It is impractical to measure the vertical displacements at all dofs of the beam. Partitioning the global beam structure into five substructures as shown in Figure 3(b), we considered the only five measurements. In the figure, the number in the square represents the substructure node. The substructure nodes are located at nodes 10, 20, 30, 40, and 50 of the beam. 


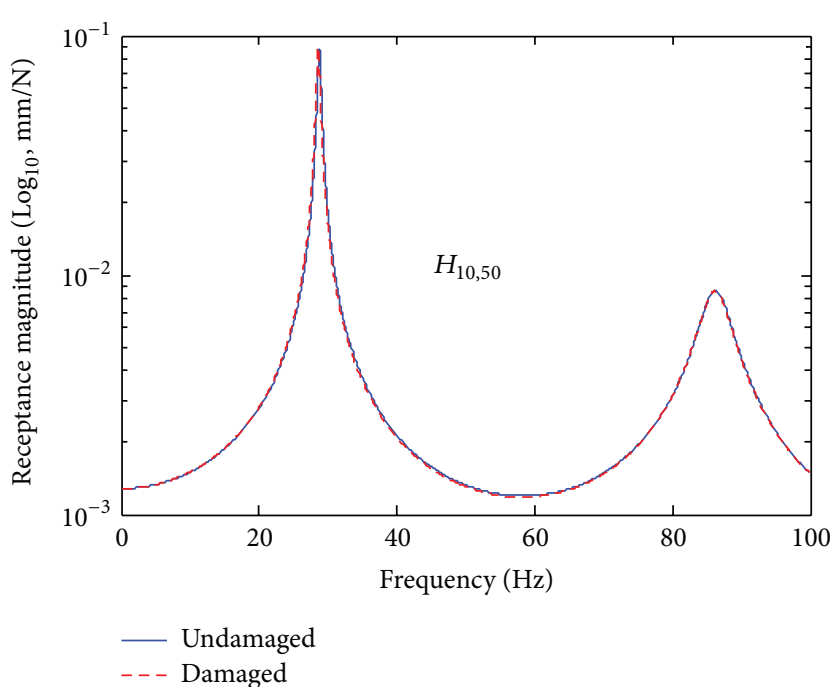

(a)

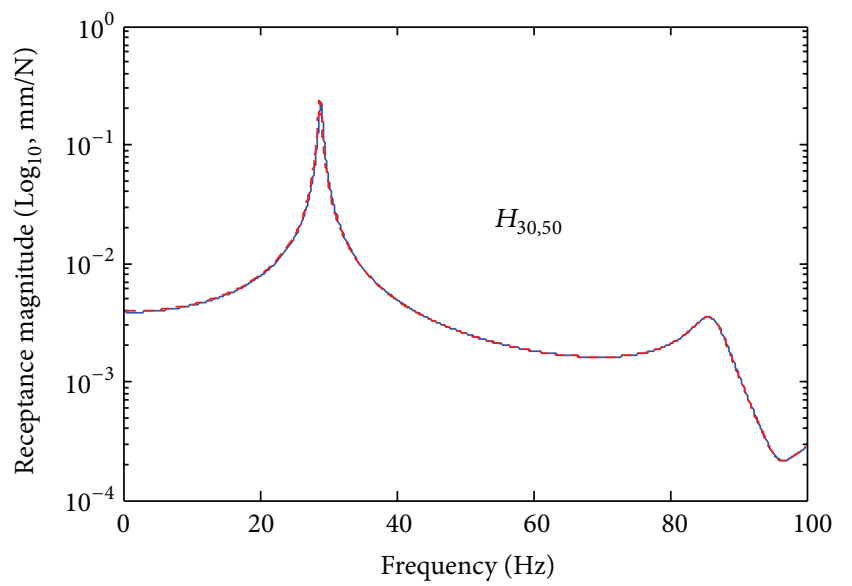

- Undamaged

_. - Damaged

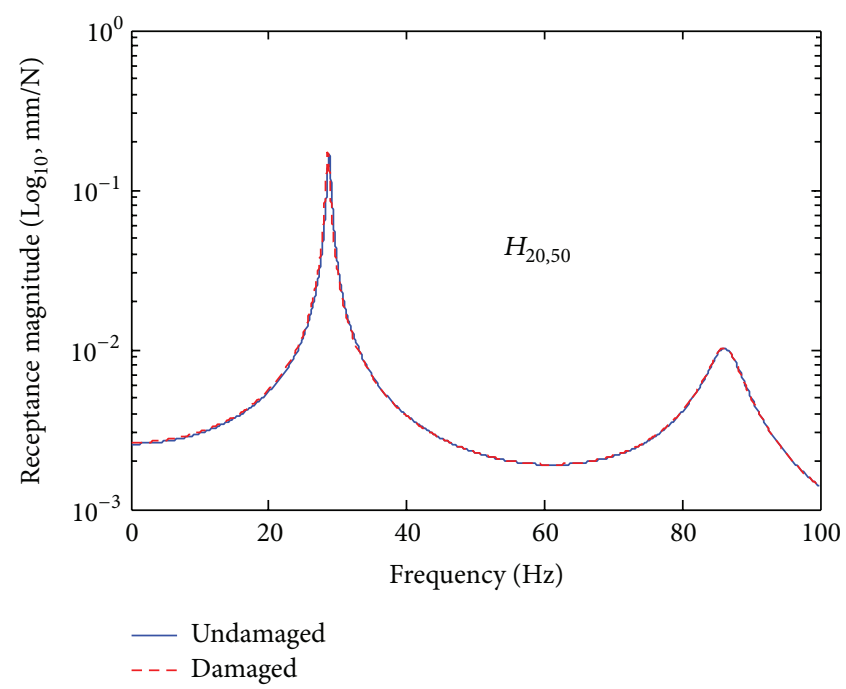

(b)

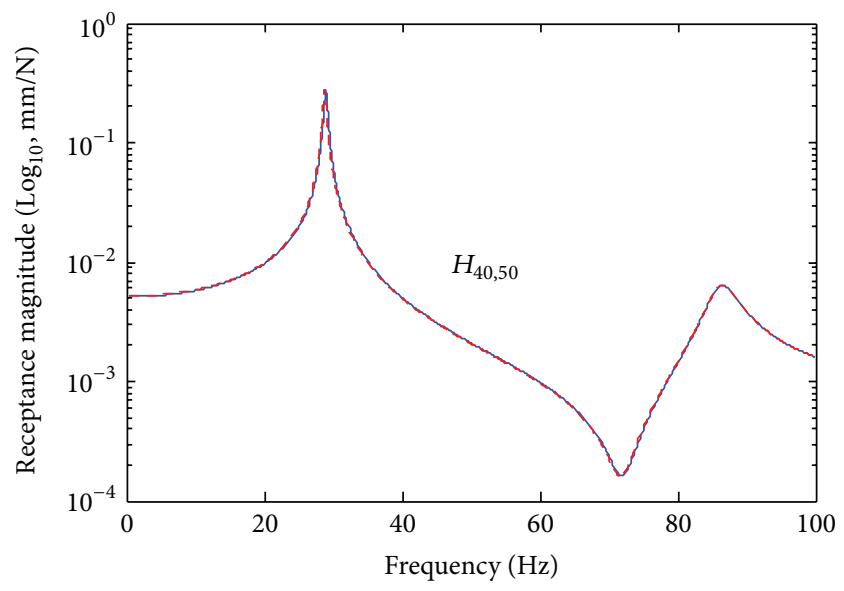

_ Undamaged

-. Damaged

(c)

(d)

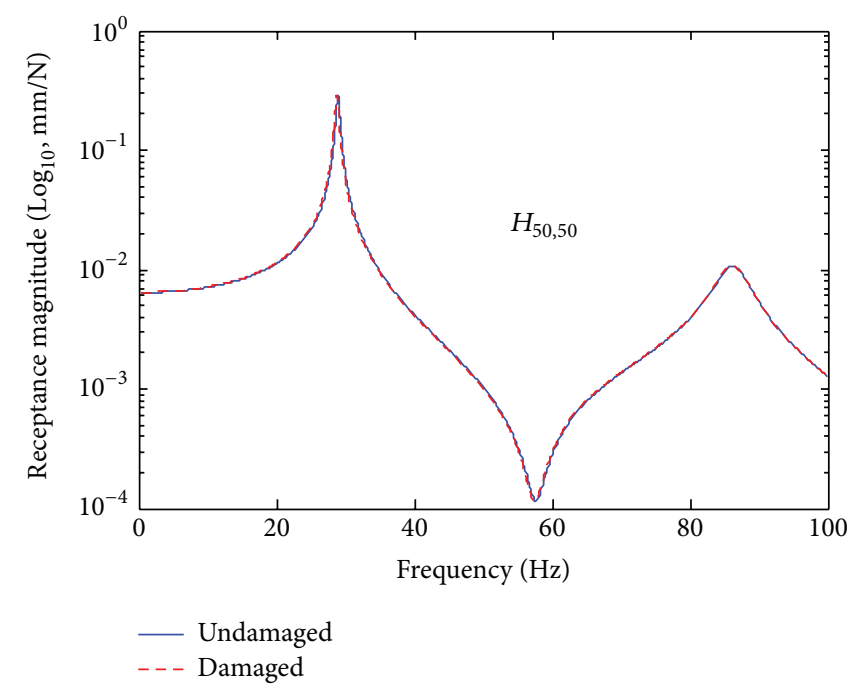

(e)

FIGURE 4: FRF curves: (a) $H_{10,50}$, (b) $H_{20,50}$, (c) $H_{30,50}$, (d) $H_{40,50}$, and (e) $H_{50,50}$. 


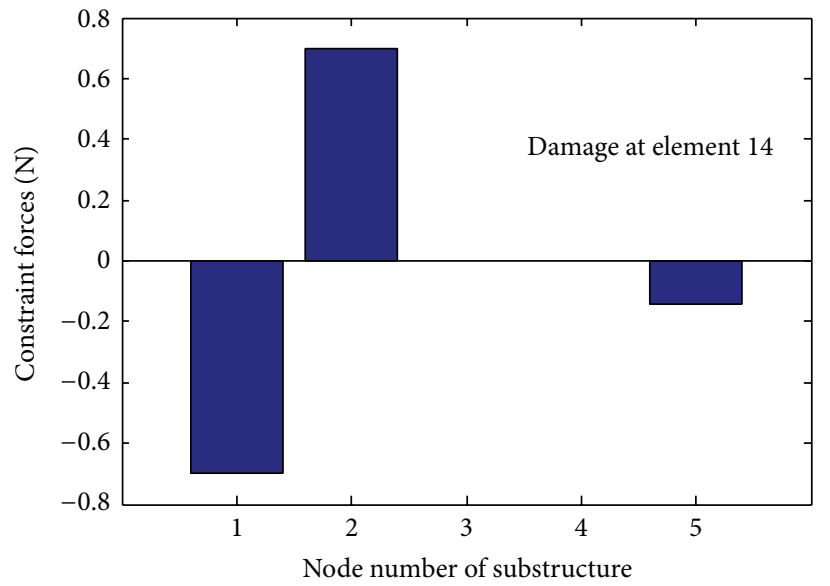

(a)

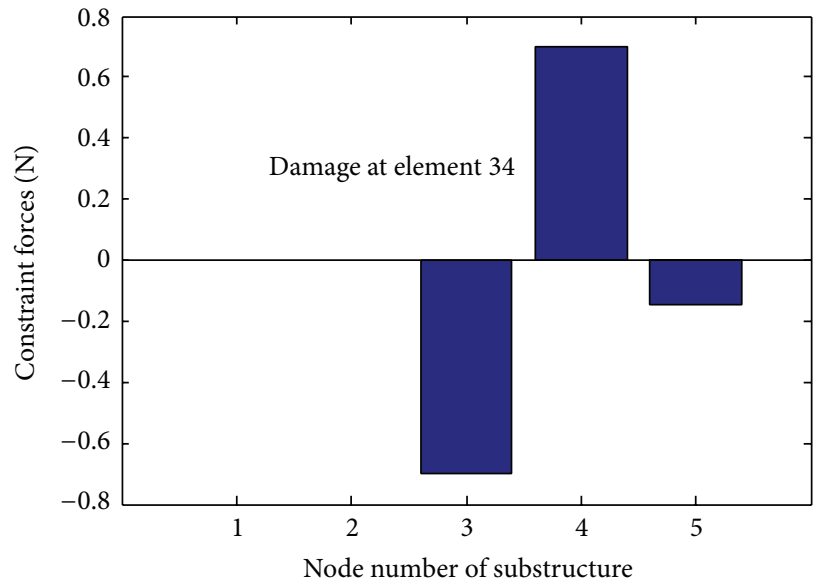

(c)

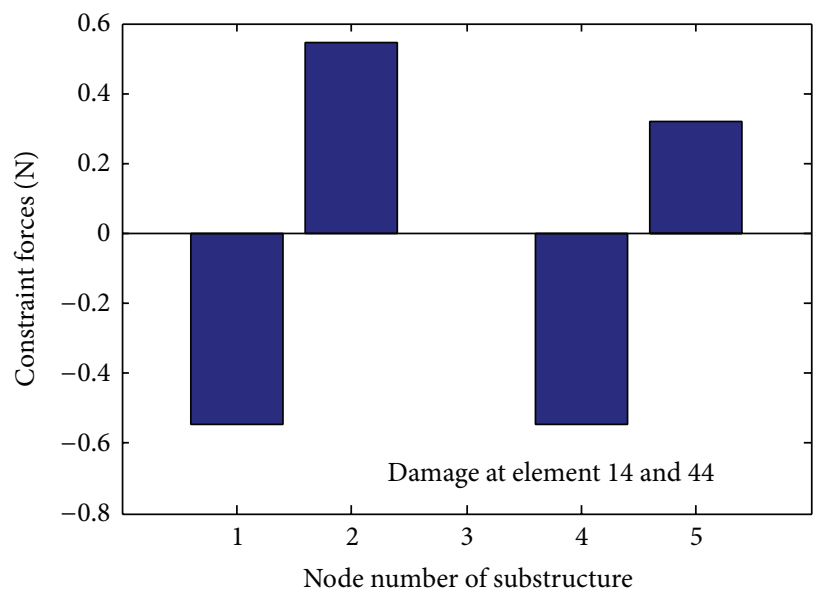

(e)

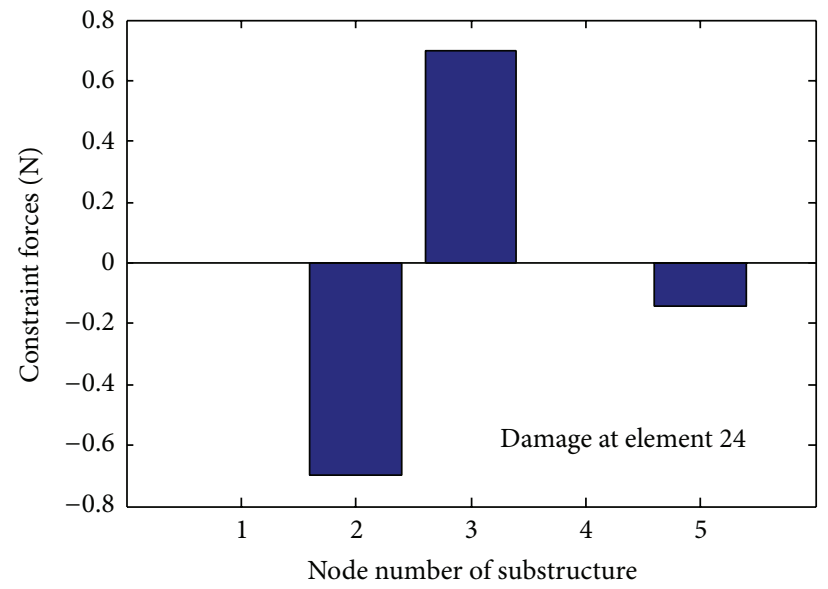

(b)

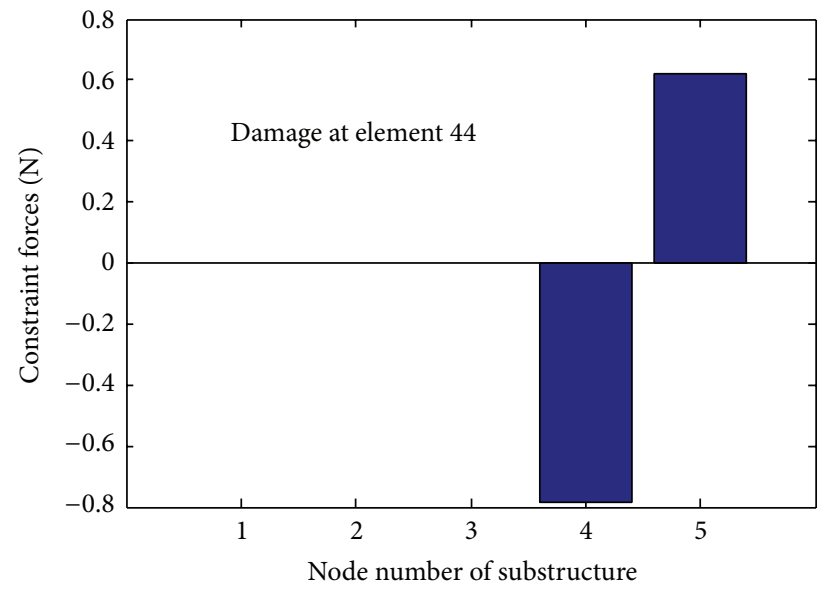

(d)

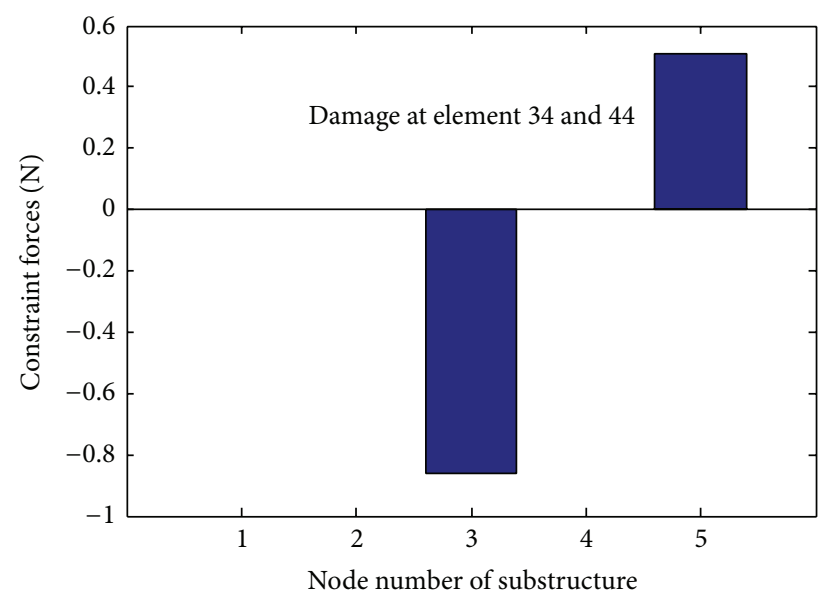

(f)

FIgURE 5: Constraint forces (noise-free): (a) damage at element 14, (b) damage at element 24, (c) damage at element 34, (d) damage at element 44, (e) damage at elements 14 and 44, and (f) damage at elements 34 and 44. 


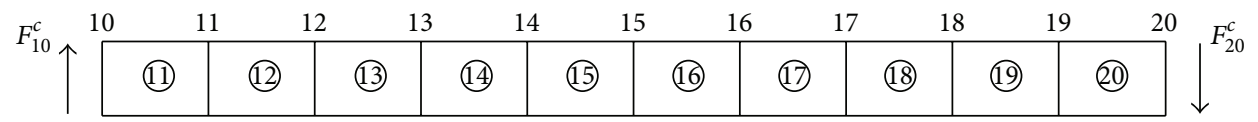

(a)

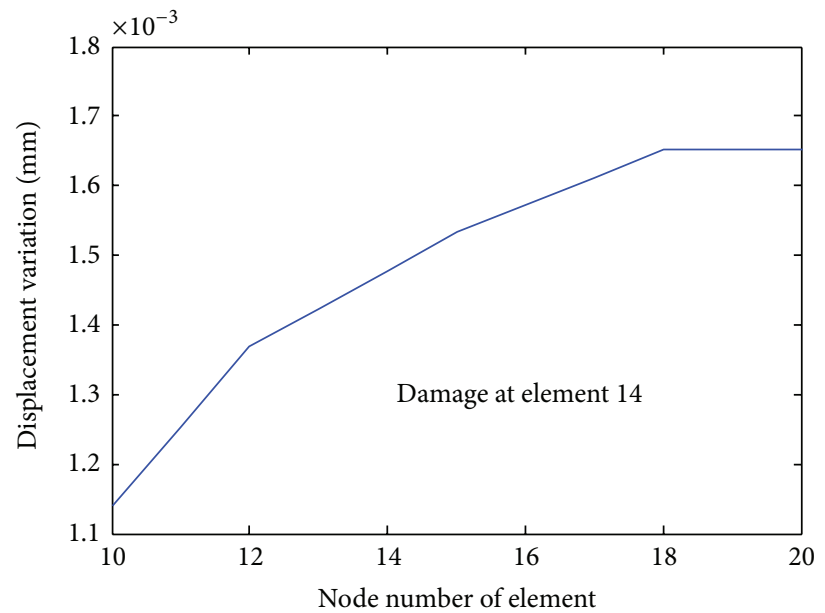

(b)

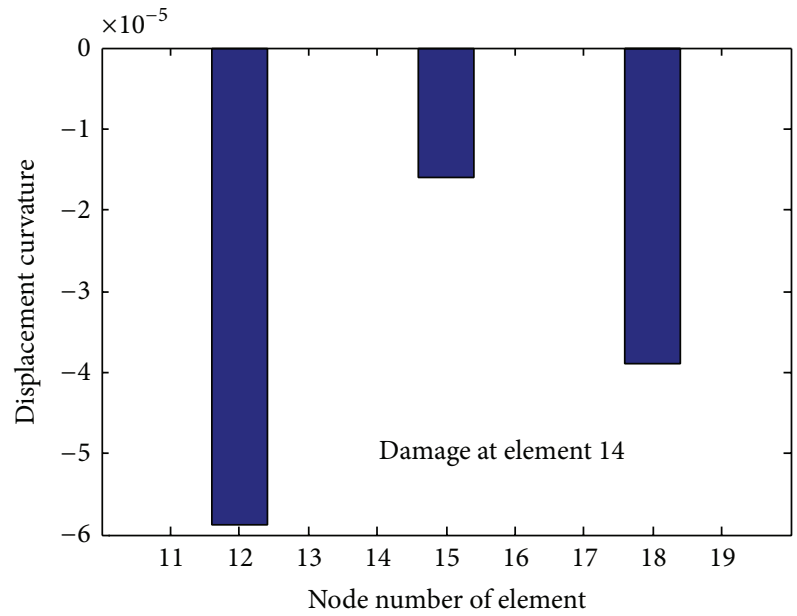

(c)

Figure 6: Damage detection of the second substructure: (a) modeling of isolated substructure, (b) displacement variation, and (c) displacement curvature.

The FRF matrix of the intact undamaged structure can be obtained from the initial dynamic parameters as

$$
\widehat{\mathbf{H}}_{R}(\Omega)=\left[\begin{array}{lllll}
\widehat{H}_{10,10} & \widehat{H}_{10,20} & \widehat{H}_{10,30} & \widehat{H}_{10,40} & \widehat{H}_{10,50} \\
\widehat{H}_{20,10} & \widehat{H}_{20,20} & \widehat{H}_{20,30} & \widehat{H}_{20,40} & \widehat{H}_{20,50} \\
\widehat{H}_{30,10} & \widehat{H}_{30,20} & \widehat{H}_{30,30} & \widehat{H}_{30,40} & \widehat{H}_{30,50} \\
\widehat{H}_{40,10} & \widehat{H}_{40,20} & \widehat{H}_{40,30} & \widehat{H}_{40,40} & \widehat{H}_{40,50} \\
\widehat{H}_{50,10} & \widehat{H}_{50,20} & \widehat{H}_{50,30} & \widehat{H}_{50,40} & \widehat{H}_{50,50}
\end{array}\right]_{R} .
$$

This numerical experiment established two damage scenarios of single and multiple damages. The single damage of $30 \mathrm{~mm}$ average length located at the elements 14, 24, 34, and 44 , respectively. The multiple damages located at the elements 14 and 44 and 34 and 44, respectively. The noise effect was investigated in both scenarios.

The damage leads to the change in the initial FRF matrix. Figure 4 represents the FRF curves of the cantilevered beam without and with a single damage at element 14 . In the plot, $H_{i, 50}(i=10,20,30,40,50)$ represents the FRF curve corresponding to the $i$ th node due to a unit impulse at node 50 . The $y$-axis in the plot represents the logarithmic scale of the receptance magnitude in the range of $0.01-100 \mathrm{~Hz}$ with the step of $0.02 \mathrm{~Hz}$. It is shown that both plots are almost the same regardless of the presence of the damage. It indicates that the damage cannot be found by the FRF plots only.

This study took the FRF data of $H_{i, 50}(i=10,20,30,40,50)$ in the narrow range of $2.05-2.2 \mathrm{~Hz}$ with the step of $0.02 \mathrm{~Hz}$ to detect damage. The frequency range was arbitrarily selected in the neighborhood of the first resonance frequency. The constraints by measured FRF data at each excitation frequency are derived as

$$
\begin{gathered}
\mathbf{A}=\left[\begin{array}{ccccc}
1 & -\frac{H_{10,50}}{H_{20,50}} & 0 & 0 & 0 \\
1 & 0 & -\frac{H_{10,50}}{H_{30,50}} & 0 & 0 \\
1 & 0 & 0 & -\frac{H_{10,50}}{H_{40,50}} & 0 \\
1 & 0 & 0 & 0 & -\frac{H_{10,50}}{H_{50,50}}
\end{array}\right], \\
\mathbf{U}_{R}=\left[\begin{array}{lllll}
U_{10, R} & U_{20, R} & U_{30, R} & U_{40, R} & U_{50, R}
\end{array}\right]^{T},
\end{gathered}
$$

where $H_{i, j}$ represents the $i$ th row and $j$ th column element in the FRF matrix at the damaged state.

The POD (proper orthogonal decomposition) is a multivariate statistical method that aims at obtaining a compact representation of the data. It is introduced in Appendix B. The POD extracts a basis to decompose the data so that the projection of the data contains as much energy as possible. The POD reduces a large number of interdependent variables to a small number of uncorrelated variables and provides relevant structure hidden in the data. The POD involves two steps of extraction of POMs (proper orthogonal modes) and the projection of basis functions to obtain a low-dimensional dynamic model. The POMs constitute a set of optimal basis functions with respect to energy content of the signal. The POM obtained from the PODs effectively extracts the principal component of a large dof system or complex physical phenomena.

Inserting (12) and (13) into (8), the constraint forces at each excitation frequency are calculated at the partitioned 


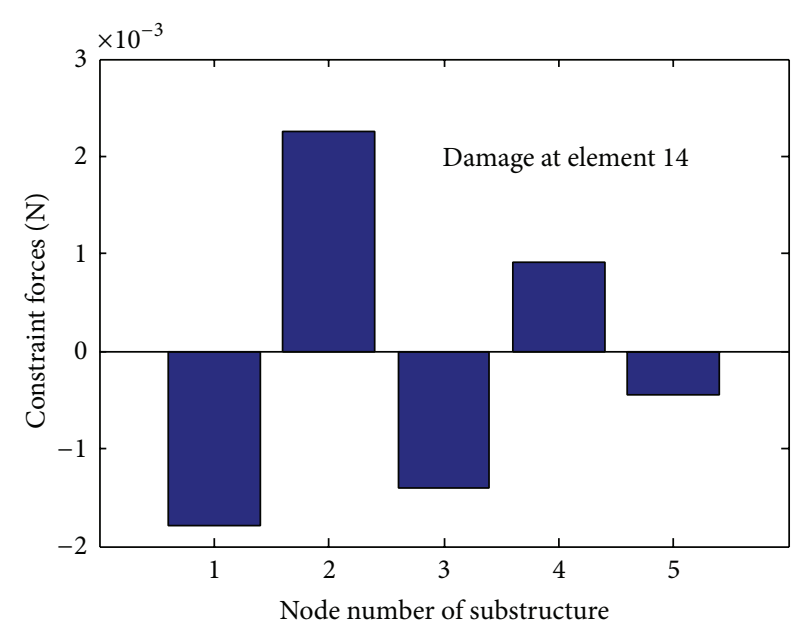

(a)

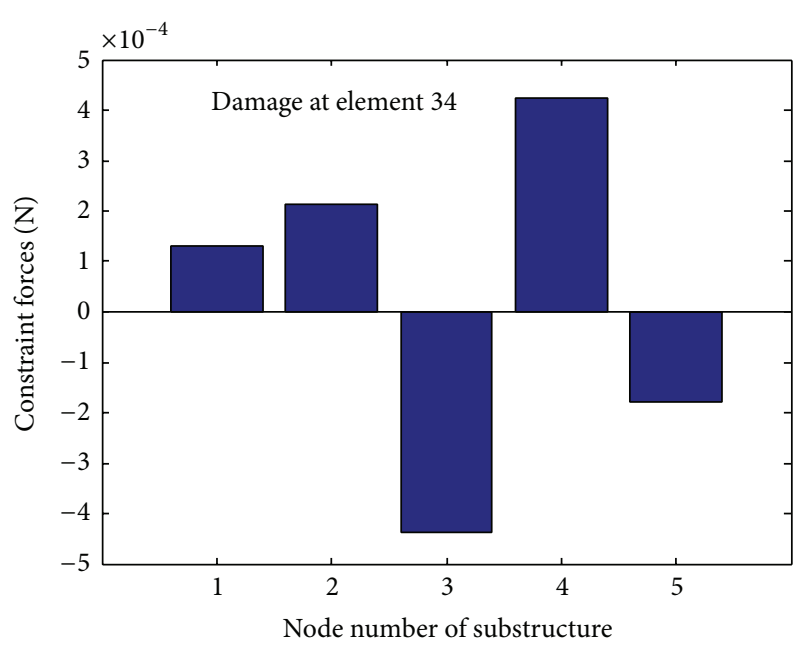

(c)

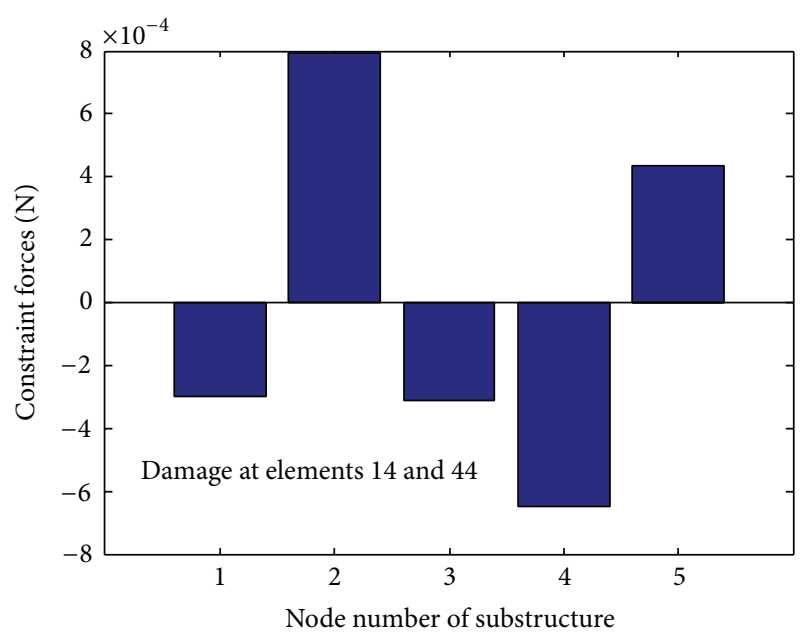

(e)

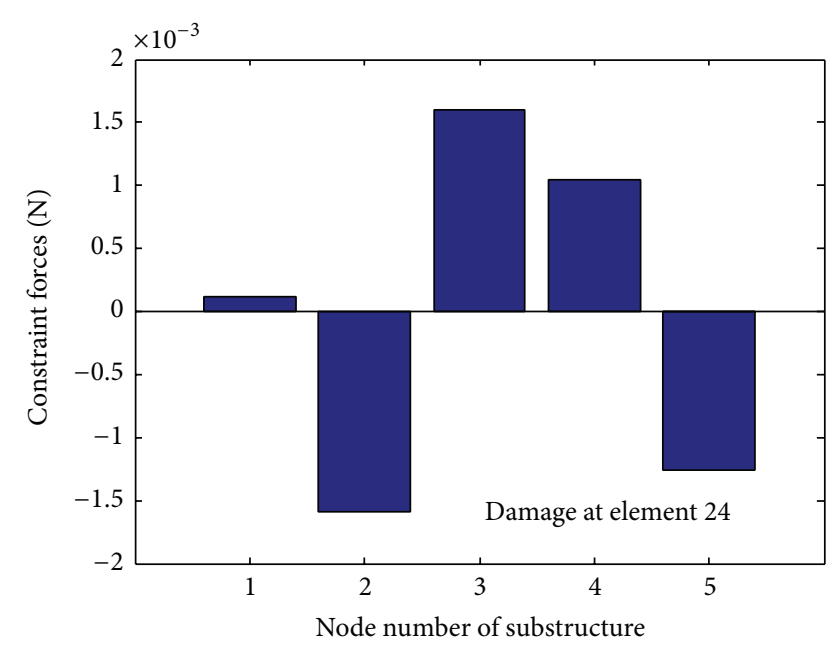

(b)

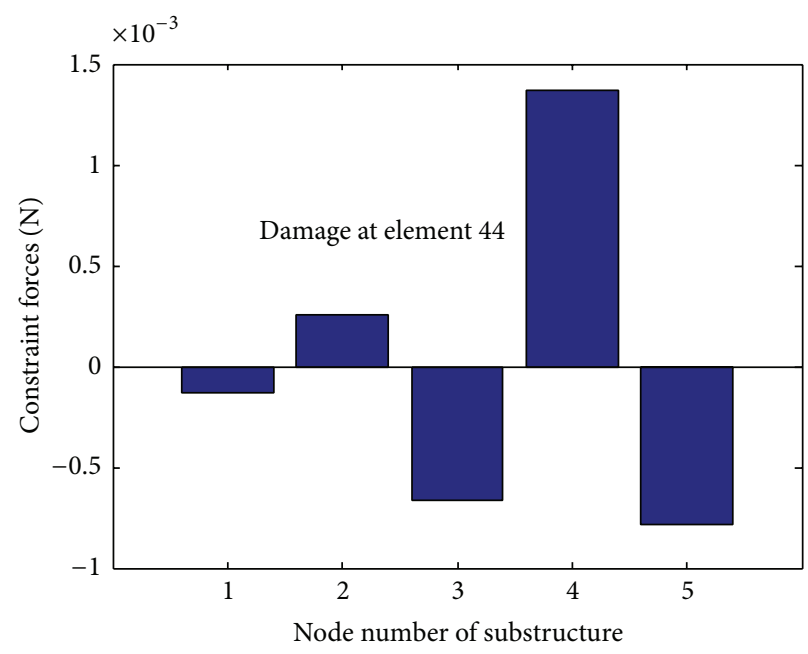

(d)

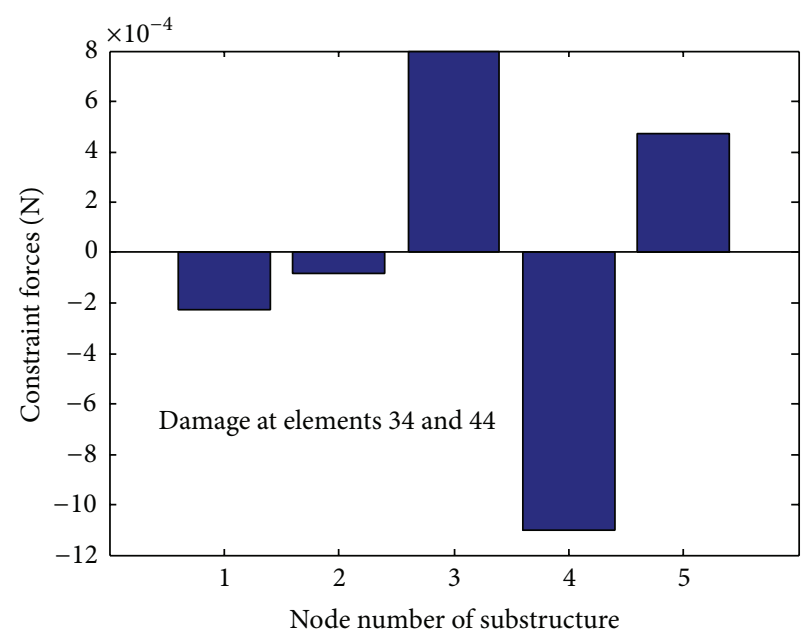

(f)

FIGURE 7: Constraint forces (1\% noise): (a) damage at element 14, (b) damage at element 24, (c) damage at element 34, (d) damage at element 44, (e) damage at elements 14 and 44, and (f) damage at elements 34 and 44. 


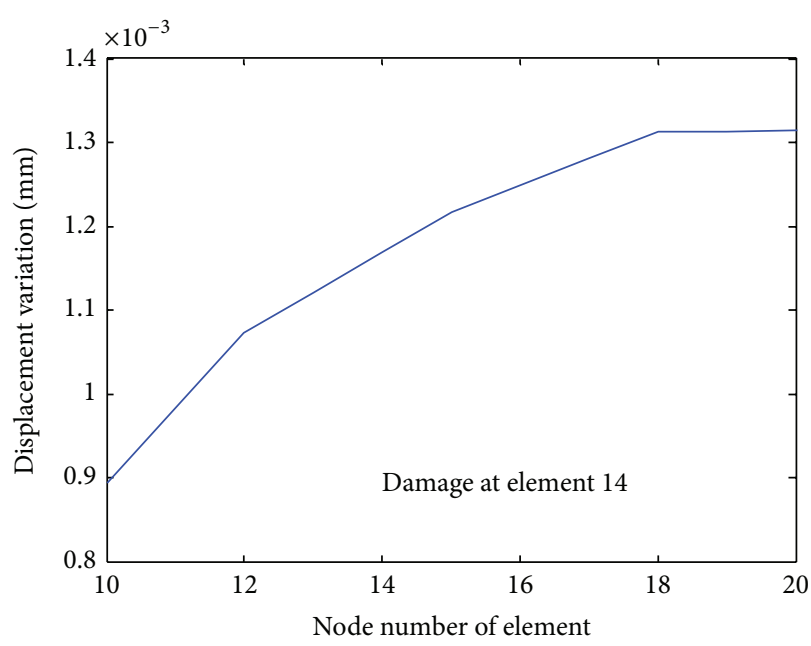

(a)

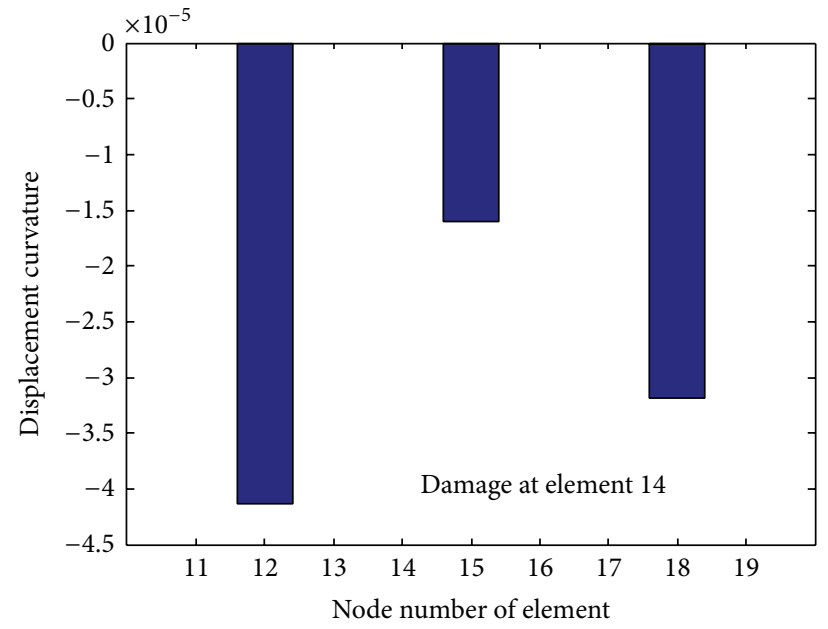

(b)

FIGURE 8: Damage detection of the isolated substructure (1\% noise): (a) displacement variation and (b) displacement curvature.

nodes. The calculated constraint forces were determined by POMs extracted from the data within the frequency range to be considered. The constraint forces based on the measured data of noise-free are plotted in Figure 5. The constraint forces represent the POM corresponding to the first POV (proper orthogonal value) of the FRF data taken within the prescribed frequency range.

As shown in the figure, it is observed that the damageexpected substructure positions between the adjacent substructure nodes to exhibit the abrupt variation of the constraint forces. Their variation results from the variation of the dynamic responses due to the damage. Such phenomenon is equivalently observed to the single-damaged and multidamaged cases. The damaged substructure can be detected by the distribution of the constraint forces calculated at the measurement positions even though the damage exists at multiple and tiny regions.

The next step is to find the damaged element from the isolated damage-expected substructure. We considered the beam damaged at element 14 . The isolated model that the damage at the substructure between nodes 10 and 20 is expected is depicted in Figure 6(a). In the figure, the constraint forces of $\mathbf{F}_{10}^{c}=0.7 \mathrm{~N}$ and $\mathbf{F}_{20}^{c}=0.7 \mathrm{~N}$ act on both ends of the isolated substructure. The substructure was modeled by 10 beam elements and 11 vertical displacements neglecting the slopes at nodes. The vertical displacements were measured at four nodes of $12,15,17$, and 18 for detecting the local damage of the substructure. Assuming the measured displacements as the constraints of (10) to describe the constrained responses of the substructure, they are expanded to the full set of displacements using (11). The displacement variation between the intact and damaged states is plotted in Figure 6(b). It is investigated that the damage can rarely be identified from the displacement curve only. If the measurement sensor does not locate at the damage location, the damage cannot be found. However, it is rarely possible to coincide the measurement location with the damage location. The displacement curvature is introduced to prevent such difficulty because it is sensitive to the region in the neighborhood of the damage. Figure 6(c) exhibits the displace-ment curvature calculated by the second-order finite differ-ence of the estimated displacements. It is observed that the abrupt variation of the displacement curvature at node 12 is caused by the damage at the element 14 . The displacement curvature indicates the damage region rather than the accurate damage location.

This application considered the effect of the noise included in the measured FRF data. The measured FRF response $H_{i, j}^{m}$ can be calculated of the simulated noise free FRF data $H_{i, j}^{0}$ as follows:

$$
H_{i, j}^{m}=H_{i, j}^{0}\left(1+\alpha \sigma_{i, j}\right)
$$

where $\alpha$ denotes the relative magnitude of the error and $\sigma_{i, j}$ is a random number variant in the range $[-1,1]$.

Figure 7 represents the constraint forces using the FRF data contaminated in $1 \%$ noise. It is shown that the damage is expected by the large variation of the constraint forces. However, it is less clear than the noise-free case. The ambiguity in detecting the damage comes from the irregular variations of the noise included in the raw FRF data. It is observed that they are sensitive to the noise.

The isolated model of the second substructure to have the damage at element 14 was considered. Combining the dynamic equation of the substructure at the intact state and the displacement constraints measured at four nodes of 12,15 , 17 , and 18 including $1 \%$ noise, the vertical displacements at 11 nodes are estimated by (11). Figure 8(a) exhibits the dynamic response but does not provide the information on the damage. Thus, the displacement data should be modified as the displacement curvature by the second-order finite difference method. Figure 8 (b) represents the displacement 
curvature. It is observed that the damage exists at the location to represent the abrupt curvature change. However, it is found that the displacement curvature is also sensitive to the noise included in the raw data. The proposed method illustrated the possibility to reduce the number of measurements. It is shown as the result of the reduction of the measurement data that the damaged substructure is detected by tracing the distribution of the constraint forces, the local damage is found by the displacement curvature, and the noise effect should be overcome.

\section{Conclusions}

This study presented a model-based substructuring method. The method is summarized by showing that the damaged substructure is detected by tracing the distribution of the constraint forces at the nodes between the partitioned substructures and the local damage is found by the displacement curvature of the isolated substructure. The constraint forces were selected as the POMs corresponding to the first POV extracted from the measured FRFs within a small range of frequency less than the first resonance. However, the method is sensitive to the noise included in the measured raw data and the noise is regarded as the obstacle in detecting the damage. A numerical application illustrated the validity of the proposed method and evaluated the noise effect.

\section{Appendices}

\section{A.}

The response of an initial structure in the frequency domain, described by $\widehat{\mathbf{U}}(\Omega)$, to an external excitation, described by $\mathbf{F}(\Omega)$, is given by

$$
\widehat{\mathbf{U}}(\Omega)=\widehat{\mathbf{H}}(\Omega) \mathbf{F}(\Omega),
$$

where $\widehat{\mathbf{H}}(\Omega)=\left(\mathbf{K}-\Omega^{2} \mathbf{M}+i \Omega \mathbf{C}\right)^{-1}$ is the FRF matrix of the initial structure, whose elements can be receptances. Equation (A.1) expresses the dynamic equation at a certain frequency $\Omega$.

Assume that the modal displacements in the frequency domain are measured as

$$
\mathbf{A U}(\Omega)=\mathbf{b}
$$

in which $\mathbf{A}$ denotes a coefficient matrix, $\mathbf{b}$ represents the measured data, and (A.2) represents constraints to govern the dynamic responses. Minimizing a quadratic form by the difference between the analytical response vector of (A.1) and the actual response vector of (A.2), the estimated response vector in the satisfaction of the constraint conditions of (A.2) can be obtained as

$$
\mathbf{U}=\widehat{\mathbf{U}}+\widehat{\mathbf{D}}^{-1 / 2}\left(\mathbf{A} \widehat{\mathbf{D}}^{-1 / 2}\right)^{+}(\mathbf{b}-\mathbf{A} \widehat{\mathbf{U}})
$$

where $\widehat{\mathbf{U}}=\widehat{\mathbf{D}}^{-1} \mathbf{F}$ and $\widehat{\mathbf{H}}^{-1}=\widehat{\mathbf{D}}$. Equation (A.3) represents the dynamic equation for describing the constrained motion.
B.

Assume that the measurement data are expressed by a finite number of points in space and frequency. Expressing a set of FRF response data as $\mathbf{U}$, suppose that $m$ linear snapshots of the response $U_{i}$ of size $n$ are obtained by response measurements written as

$$
\begin{aligned}
\mathbf{U} & =\left[\begin{array}{llll}
\mathbf{U}^{(1)} & \mathbf{U}^{(2)} & \cdots & \mathbf{U}^{(m)}
\end{array}\right] \\
& =\left[\begin{array}{cccc}
U_{1}^{(1)} & U_{1}^{(2)} & \cdots & U_{1}^{(m)} \\
U_{2}^{(1)} & U_{2}^{(2)} & \cdots & U_{2}^{(m)} \\
\vdots & \vdots & \ddots & \vdots \\
U_{n}^{(1)} & U_{n}^{(2)} & \cdots & U_{n}^{(m)}
\end{array}\right],
\end{aligned}
$$

where the superscript $(j)$ represents the FRF frequency response data at the $j$ th frequency $\Omega_{j}, n$ denotes the number of measurement positions of the structure, and $m$ is the total number of frequency observations.

The POD of this discrete field consists in solving the eigenvalue problem. Let $m \times m$ matrix $\widetilde{\mathbf{C}}$ be defined as

$$
\widetilde{\mathbf{C}}=\mathbf{U}^{T} \mathbf{U}
$$

Solving the eigenvalue problem of (9) at the core of the POD method, it satisfies

$$
\widetilde{\mathbf{C}} \varphi_{k}=\lambda_{k} \varphi_{k}, \quad k=1, \ldots, m,
$$

where the eigenvalues are arranged as

$$
\lambda_{1} \geq \lambda_{2} \geq \cdots \geq \lambda_{m} \geq 0,
$$

where the eigenvalues $\lambda_{k}$ are the proper orthogonal values (POVs) and each eigenvector $\varphi_{k}$ of the extreme value problem is associated with a POV $\lambda_{k} \cdot \varphi_{1}$ represents the eigenvector corresponding to the largest eigenvalue $\lambda_{1}$. If the eigenvalues are normalized, the relative energy captured by the corresponding POM.

The POMs may be used as a basis for the decomposition of $\mathrm{U}$. The POM associated with the greatest POV is the optimal vector. If the eigenvalues are normalized, they represent the relative energy captured by the corresponding POM. The eigenvalue reflects relative kinetic energy associated with the corresponding mode. The energy is defined as the sum of the POVs. The POMs are written as

$$
\psi^{k}=\frac{\sum_{i=1}^{m} \boldsymbol{\varphi}_{i}^{k} \mathbf{U}^{(i)}}{\left\|\sum_{i=1}^{m} \boldsymbol{\varphi}_{i}^{k} \mathbf{U}^{(i)}\right\|}, \quad k=1, \ldots, m .
$$

And the POMs are arranged as

$$
\psi=\left[\begin{array}{llll}
\psi^{1} & \psi^{2} & \cdots & \psi^{m}
\end{array}\right]
$$

\section{Conflict of Interests}

The authors declare that there is no conflict of interests regarding the publication of this paper. 


\section{Acknowledgment}

This work was supported by the National Research Foundation of Korea (NRF) Grant funded by the Korea government (MEST) (no. 2011-0012164).

\section{References}

[1] J. Hou, Ł. Jankowski, and J. Ou, "Local damage identification in frequency domain based on substructure isolation method," in Proceedings of the 6th International Workshop on Advanced Smart Materials and Smart Structures Technology (ANCRiSST '11), pp. 25-26, 2011.

[2] Q. W. Yang, "A new damage identification method based on structural flexibility disassembly," Journal of Vibration and Control, vol. 17, no. 7, pp. 1000-1008, 2011.

[3] Q. W. Yang and B. X. Sun, "Structural damage localization and quantification using static test data," Structural Health Monitoring, vol. 10, no. 4, pp. 381-389, 2011.

[4] K. V. Yuen and L. S. Katafygiotis, "Substructure identification and health monitoring using noisy response measurements only," Computer-Aided Civil and Infrastructure Engineering, vol. 21, no. 4, pp. 280-291, 2006.

[5] S. S. Law, K. Zhang, and Z. D. Duan, "Structural damage detection from coupling forces between substructures under support excitation," Engineering Structures, vol. 32, no. 8, pp. 2221-2228, 2010.

[6] Z. Xing and A. Mita, "A substructure approach to local damage detection of shear structure," Structural Control and Health Monitoring, vol. 19, no. 2, pp. 309-318, 2012.

[7] J. T. Xing, W. G. Price, and Z. H. Wang, "A substructure approach to power flow analysis and application to engineering structures," in Proceedings of the 8th International Conference on Recent Advances in Structural Dynamics, Paper Number 101, Southampton, UK, July 2003.

[8] Y. Lei, C. Liu, and L. J. Liu, "A two stage damage detection method with application to the phrase I ASCE SHM benchmark building," in Proceedings of the 12th International Conference on Engineering, Science, Construction, and Operations in Challenging Environments, pp. 2277-2288, Honolulu, Hawaii, USA, March 2010.

[9] J. Hou, Ł. Jankowski, and J. Ou, "Substructure isolation and identification using FFT of measured local responses," in Proceedings of the 5th European workshop on Structural Health Monitoring (EWSHM '10), 2010.

[10] J. Qian, X. Ji, W. Zhang, L. Xu, X. Fu, and L. Gu, “Damage detection test of a substructure model of the National Swimming Center," Science in China E, vol. 51, no. 7, pp. 940-948, 2008.

[11] D. Zhang and E. A. Johnson, "Substructure identification for shear structures: cross-power spectral density method," Smart Materials and Structures, vol. 21, no. 5, Article ID 055006, 2012.

[12] S. Law, K. Zhang, and Z. Duan, "Structural damage detection from coupling forces between substructures," in Proceedings of the 15th International Congress on Sound and Vibration, 2008.

[13] S. Weng, Y. Xia, and Y. L. Xu, "Model updating with improved substructuring method," in Proceedings of the 3rd International Conference on Integrity, Reliability and Failure, 2009.

[14] S. Rahmatalla, H. C. Eun, and E. T. Lee, "Damage detection from the variation of parameter matrices estimated by incomplete FRF data," Smart Structures and Systems, vol. 9, no. 1, pp. 55-70, 2012. 

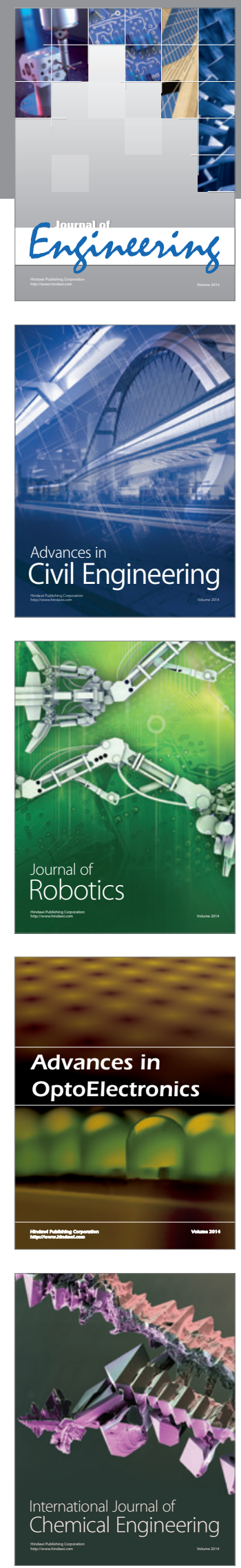

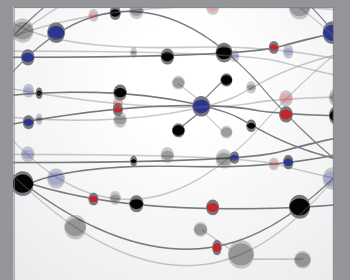

The Scientific World Journal
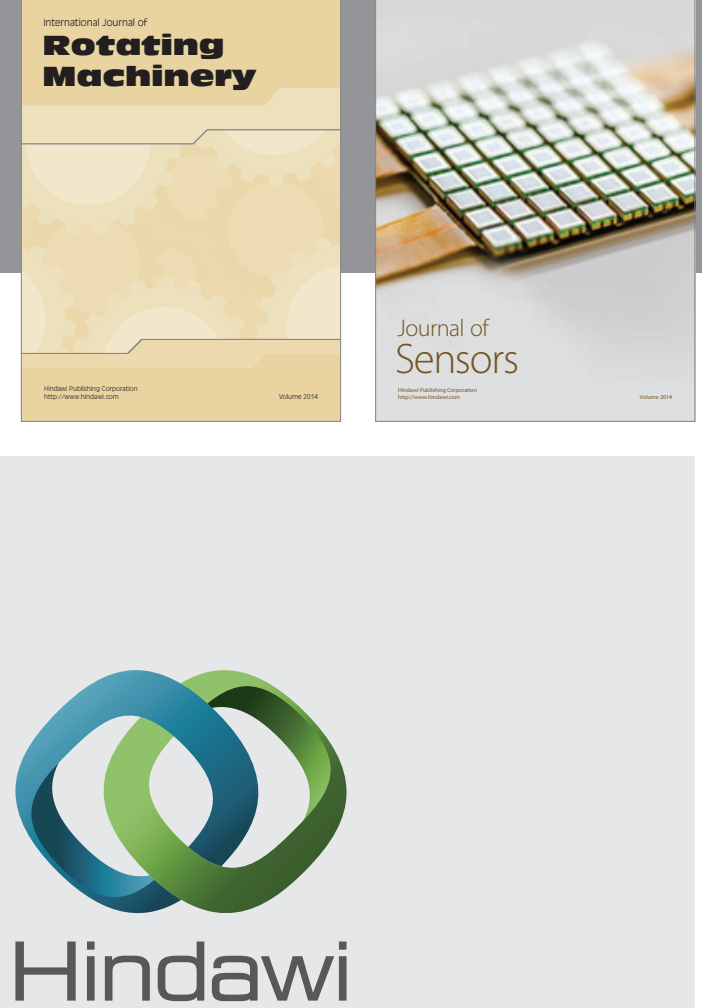

Submit your manuscripts at http://www.hindawi.com
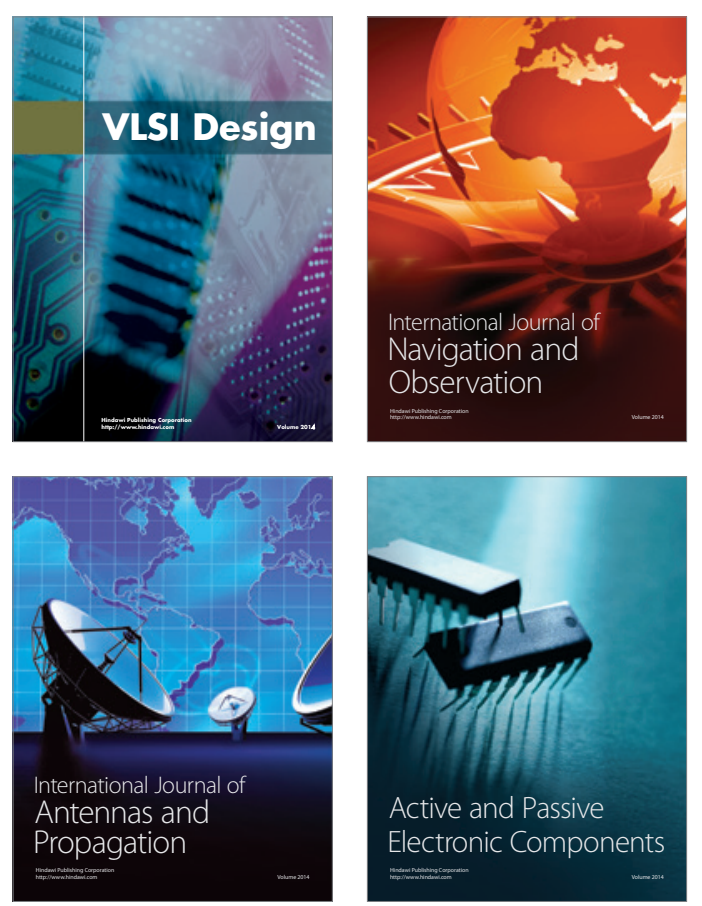
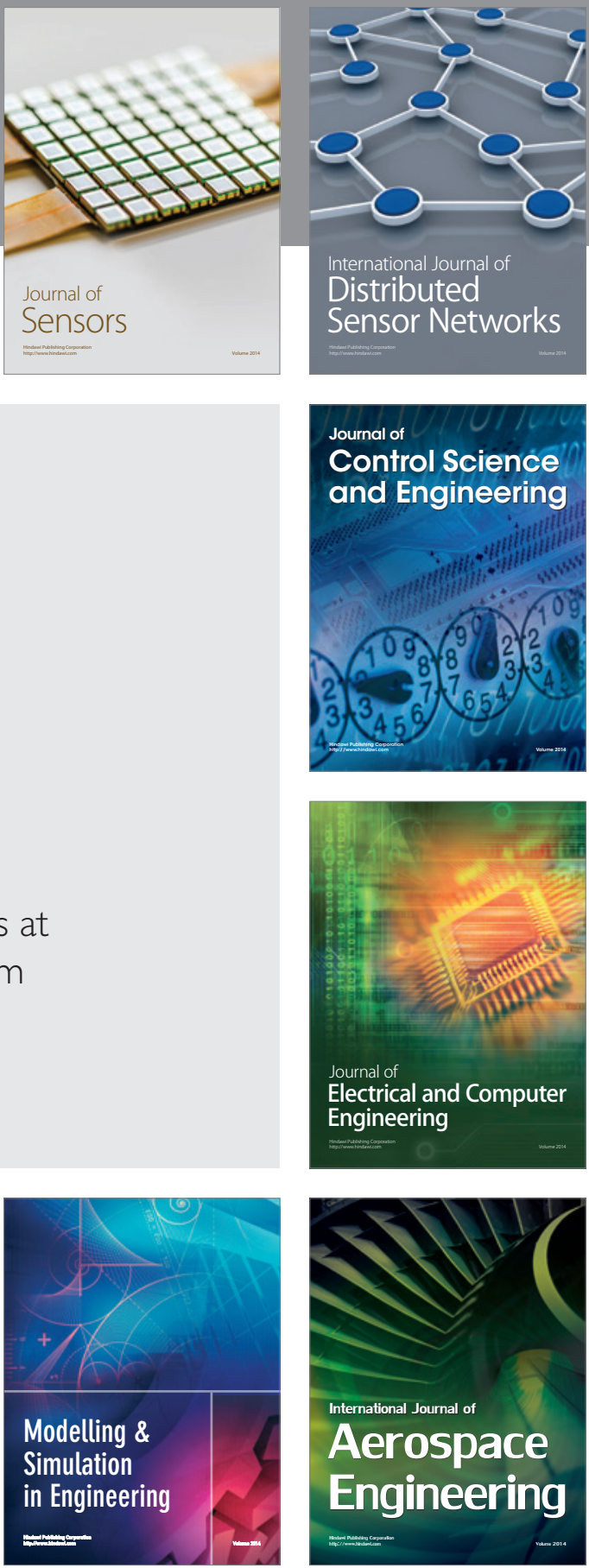

Journal of

Control Science

and Engineering
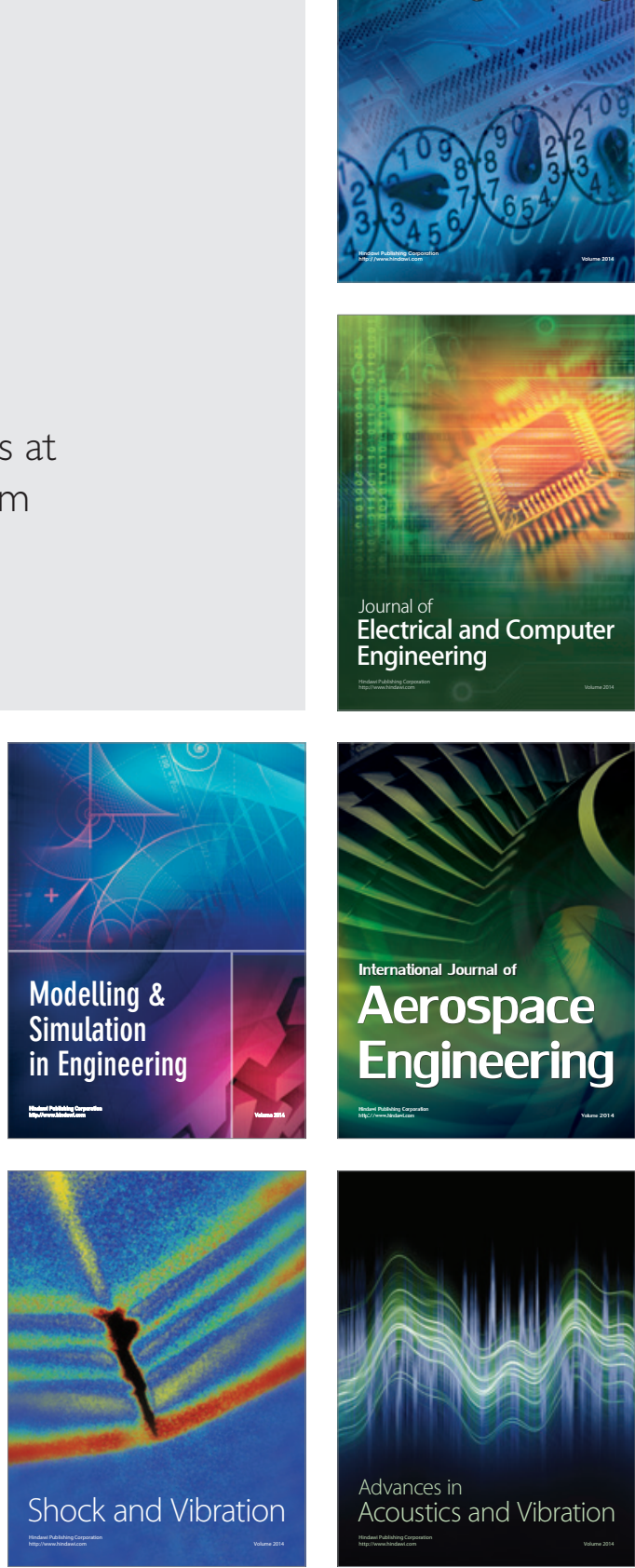